\title{
Acute Abdominal Pain Assessment in the Emergency Department: The Experience of a Greek University Hospital
}

\author{
Dimitrios Velissaris ${ }^{\mathrm{a}}$, Menelaos Karanikolas ${ }^{\mathrm{b}, \mathrm{f}}$, Nikolaos Pantzaris ${ }^{\mathrm{c}}$, George Kipourgos ${ }^{\mathrm{d}}$, \\ Vasileios Bampalis ${ }^{\mathrm{d}}$, Konstantina Karanikola ${ }^{\mathrm{d}}$, Eleftheria Fafliora ${ }^{\mathrm{d}}$, \\ Christina Apostolopoulouc ${ }^{\mathrm{c}}$, Charalampos Gogos ${ }^{\mathrm{a}}$
}

\begin{abstract}
Background: Acute abdominal pain (AAP) is a common symptom in the emergency department (ED). Because abdominal pain can be caused by a wide spectrum of underlying pathology, evaluation of abdominal pain in the ED requires a comprehensive approach, based on patient history, physical examination, laboratory tests and imaging studies. The aim of this study was to investigate predictive factors for admission to the hospital in patients who presented to the ED with AAP as the main symptom.
\end{abstract}

Methods: This prospective observational study enrolled 125 patients who presented with AAP in the ED of the Patras University Hospital in western Greece. The sample of patients who enrolled in the study was representative of patients who receive care in this academic institution. All patients underwent clinical examination, laboratory testing and radiological assessment. Clinical and laboratory data were analyzed in an attempt to identify clinical or laboratory factors predicting hospital admission.

Results: Based on clinical, laboratory and radiologic evaluation, $37.6 \%$ of patients enrolled in the study were admitted to the hospital, whereas $62.4 \%$ were not admitted. Compared to patients who were not admitted, patients admitted to the hospital had higher age and significantly higher inflammatory markers, white blood count and Creactive protein (CRP). Binary logistic regression analysis showed that abnormal imaging findings (odds ratio $(\mathrm{OR})=6.47,95 \%$ confidence interval $(\mathrm{CI}): 2.11-19.77, \mathrm{P}<0.001)$ and elevated serum CRP levels $(\mathrm{OR}=6.24,95 \% \mathrm{CI}: 2.16-18.03, \mathrm{P}<0.001)$ were significant predictive factors for hospital admission.

Manuscript submitted September 18, 2017, accepted October 9, 2017

${ }^{a}$ Internal Medicine Department, University of Patras, University Hospital of Patras, Greece

${ }^{b}$ Department of Anesthesiology, Washington University School of Medicine, St. Louis, MO 63144, USA

cUniversity of Patras, Greece

dEmergency Department, University Hospital of Patras, Greece

${ }^{\mathrm{f}}$ Corresponding Author: Menelaos Karanikolas, Department of Anesthesiology, Washington University School of Medicine, 660 S. Euclid Ave, Campus Box 8054, St. Louis, MO 63144, USA.

Email: menelaos.karanikolas@gmail.com

doi: https://doi.org/10.14740/jocmr3206w
Conclusions: Assessment of AAP remains a challenging problem in the ED. Comprehensive history combined with detailed clinical examination, appropriate laboratory testing and radiologic imaging facilitates effective assessment of patients who present in the ED with AAP and guides the decision to admit patients to the hospital for further care.

Keywords: Acute abdominal pain; Emergency room; Hospital admission; Diagnosis; Physical examination

\section{Introduction}

Abdominal pain is one of the most common reasons for patient visits in the emergency department (ED) of tertiary care hospitals, and differential diagnosis in such cases is broad because of the wide spectrum of possible underlying pathologies. Assessment of patients with abdominal pain in the ED can be challenging and is based on patient history, combined with clinical, laboratory and radiological evaluation. Because timely evaluation of acute abdominal pain (AAP) may determine prognosis, emergency room physicians must consider several diagnoses and complete patient evaluation after taking into account all available clinical, laboratory and radiologic findings in a timely fashion, in an attempt to reduce morbidity and mortality [1-3]. It is worth noting that certain populations, such as elderly and immunocompromised patients can present with atypical signs and symptoms, and therefore evaluation of abdominal pain in these patients can be more difficult [4]. The aim of this study was to record data from patients with AAP who presented to the ED of a university hospital in western Greece, in an attempt to determine predictive factors for admission to the hospital.

\section{Materials and Methods}

\section{Study population and design}

This was a prospective observational study on 125 patients who presented with AAP in the ED of the University Hospi- 
tal of Patras, Greece, in two time periods: October 1 through November 30, 2015 and April 15 through May 31, 2016. Patients were eligible to participate if they presented to the ED with AAP as the main symptom, and the study protocol was approved by the Hospital Research and Ethics Committee. Depending on patient's condition, verbal consent for participation in the study was obtained from each patient or a designated family member, and was documented in writing by two researchers. Data were de-identified and stored in an electronic database in a secure, locked computer. The research team included consultants and resident physicians, staff nurses and medical students: consultants and resident physicians assessed patients and directed patient care, while medical students collected data.

\section{Data collection}

For the purposes of this study, physicians and medical students completed a data form, which included information from the history, physical examination, laboratory tests and radiological imaging studies for each patient. Data collected included age, gender, past medical history, detailed information about pain, including pain onset, intensity (mild, moderate, and severe), character (continuous and colicky), location, migration, exacerbating and relieving factors, together with accompanying symptoms such as vomiting, constipation, diarrhea, hematemesis, melena, macroscopic hematuria, tachycardia, fever, anorexia, shivering, jaundice and rash. Data from physical examination of the abdomen included tenderness, location at palpation, evaluation of bowel sounds (normal, increased, and decreased), specific signs such as Murphy, McBurney, Psoas and rebound tenderness, rectal examination and vital signs. Laboratory test results, X-ray, ultrasound (U/S) and computerized axial tomography (CAT) scan data were also collected. Diagnosis in the ED, decision to admit the patient or not, and the ward where each patient was admitted were also recorded.

\section{Statistical analysis}

Data were analyzed using descriptive statistical tools, including frequencies for discrete variables or mean and standard deviation for continuous variables. Data analysis included Pearson's Chi-square test for associations between categorical variables, independent sample $t$-tests for comparisons between groups and bivariate correlation analysis using Pearson's coefficient. Binomial logistic regression was also performed in an attempt to identify factors predicting hospital admission. All data analysis was conducted using the SPSS Statistics version 17.0 software package, except for Chisquare and Fisher's exact tests, which were conducted using the StatCalc component of the Epi Info statistical software package, which is freely available from the Centers for Disease Control (CDC). In order to avoid concerns about false positive findings due to multiple comparisons, $\mathrm{P}$ values for significance were adjusted to more stringent values using the Bonferroni correction.

\section{Results}

\section{Demographics}

Minimum patient age was 15 years and maximum age was 96 years (mean 46.16 years). Mean age was significantly higher in patients admitted to the hospital, compared to those not admitted $(51.5 \pm 23.7$ vs. $43.0 \pm 18.3, \mathrm{P}<0.044) .34 .4 \%$ of patients were males and $65.6 \%$ were females, and there was no significant association between gender and hospital admission.

\section{Pain location and characteristics}

Pain onset was within the last $24 \mathrm{~h}$ prior to arrival in the ED in $62.4 \%$ of patients, within the last $1-5$ days in $28 \%$ and more than 5 days before arrival to the ED in $9.6 \%$ of patients. With regards to pain intensity, $13.6 \%$ of patients reported mild pain, $44.8 \%$ reported moderate pain and $41.6 \%$ reported intense pain. Pain was described as continuous in $56.8 \%$ of the patients and colicky in 36\%.

Most common pain location was in the epigastrium in $39.2 \%$ of cases, followed by diffuse pain in $18.4 \%$, right upper abdominal quadrant pain in $15.2 \%$, pain in the hypogastrium in $13.6 \%$, right and left iliac fossa pain in $12.8 \%$ and left upper abdominal quadrant pain in $4.8 \%$ of patients.

\section{Associated symptoms and physical findings}

Vomiting was the most common accompanying symptom, and was reported by $32.8 \%$ of patients, whereas diarrhea was present in $16 \%$, constipation in $12 \%$, fever in $18.4 \%$ and loss of appetite in $21.6 \%$ of patients (Table 1). Patients admitted to the hospital had significantly higher incidence of vomiting (23 of 47 vs. 18 of $78, \mathrm{P}<0.003$ ) and fever $(17$ of 47 vs. 6 of $78, \mathrm{P}<0.001)$ compared to patients not admitted. In addition, admitted patients had higher frequency of tachycardia, diarrhea and anorexia, but differences were not significant. Pain intensity was also associated with hospital admission, so that the likelihood of hospital admission was significantly higher in patients with increasing pain intensity $(\mathrm{P}<0.018)$. Furthermore, certain physical examination signs had higher prevalence in admitted patients: Murphy sign ( 7 of 47 vs. 3 of 78, $\mathrm{P}<0.041$ ), McBurney sign (11 of 47 vs. 2 of $78, \mathrm{P}$ $<0.001)$ and rebound tenderness (16 of 47 vs. 2 of $78, \mathrm{P}<$ $0.001)$. Frequency of physical and radiologic findings was compared in patients admitted to the hospital vs. patients not admitted to the hospital using the Chi-square or Fisher's exact test as appropriate. However, after adjusting $\mathrm{P}$ values for significance to $0.05 / 16=\mathrm{P}<0.031$ using Bonferroni correction, only differences in McBurney sign and rebound tenderness remained significant between groups. Surgical consultation was requested in $47(37.6 \%)$ patients, and the likelihood of hospital admission was significantly higher (31 of 47 vs. 16 of $78, \mathrm{P}<0.001)$ in these patients.

Data on pain intensity, physical and radiologic findings 
Table 1. Vital Signs, Pain Intensity, Other Symptoms, Physical Findings, Radiologic Studies and Surgical Consultation in Patients Admitted vs. Patients Not Admitted to the Hospital

\begin{tabular}{llll}
\hline & Admitted $(\mathbf{n}=\mathbf{4 7})$ & Not admitted $(\mathbf{n}=\mathbf{7 8})$ & P value \\
\hline Systolic arterial pressure & $132.3 \pm 22.3$ & $128.4 \pm 20.6$ & 0.319 \\
Diastolic arterial pressure & $73.2 \pm 13.0$ & $128.4 \pm 20.6$ & 0.813 \\
Temperature & $36.8 \pm 0.7$ & $36.5 \pm 0.5$ & 0.015 \\
Pain intensity & & & 0.018 \\
$\quad$ Mild $(\mathrm{n}=17)$ & $4(8.5 \%)$ & $13(16.7 \%)$ & 0.041 \\
$\quad 17(36.2 \%)$ & $39(50.0 \%)$ & 0.001 \\
$\quad 26(55.3 \%)$ & $26(33.3 \%)$ & 0.001 \\
$\quad$ Moderate $(\mathrm{n}=56)$ & $7(14.9 \%)$ & $3(3.8 \%)$ & 0.003 \\
Murphy sign $(\mathrm{n}=10)$ & $11(23.4 \%)$ & $2(2.6 \%)$ & 0.001 \\
McBurney sign $(\mathrm{n}=13)$ & $16(34.0 \%)$ & $2(2.6 \%)$ & 0.026 \\
Rebound tenderness $(\mathrm{n}=18)$ & $23(48.9 \%)$ & $18(23.1 \%)$ & 0.001 \\
Vomiting $(\mathrm{n}=41)$ & $17(36.2 \%)$ & $6(7.7 \%)$ & 0.001 \\
Fever $(\mathrm{n}=23)$ & $7(14.9 \%)$ & $2(2.5 \%)$ & 0.070 \\
Tachycardia $(\mathrm{n}=9)$ & $31(65.9 \%)$ & $16(20.5 \%)$ & 0.055 \\
Surgical consultation $(\mathrm{n}=47)$ & $42(89.4 \%)$ & $42(63.8 \%)$ & 0.001 \\
Chest X-ray $(\mathrm{n}=84)$ & $33(70.2 \%)$ & $42(63.8 \%)$ & 0.001 \\
\hline Abdomen X-ray $(\mathrm{n}=75)$ & $27(57.4 \%)$ & $31(39.7 \%)$ & $6(7.7 \%)$ \\
\hline US abdomen $(\mathrm{n}=58)$ & $15(31.9 \%)$ & $23(29.5 \%)$ & \\
CAT abdomen $(\mathrm{n}=21)$ & $35(77.5 \%)$ & & \\
Image findings $(\mathrm{n}=58)$ & & & \\
\hline
\end{tabular}

Due to multiple comparisons, only $\mathrm{P}$ values $0.05 / 16$, therefore $\mathrm{P}<0.0031$ are considered significant.

and surgical consultation are summarized in Table 1.

\section{Vital signs and laboratory results}

Laboratory variables were compared between patients who did vs. patients who did not get admitted to the hospital using Student's $t$-test for independent variables, and the $\mathrm{P}$ value for significance was adjusted to $0.05 / 21=0.0023$ using the Bonferroni correction, in order to avoid concerns about false positive findings due to multiple comparisons. Data analysis showed that, compared to patients who were not admitted, patients admitted to the hospital had significantly higher white blood cells (WBCs) $(14,193 \pm 6,182$ vs. $9,641 \pm 2,883, \mathrm{P}<0.001)$, higher neutrophil count $(79.99 \pm 9.21$ vs. $67.72 \pm 12.57, \mathrm{P}<0.001)$, lower lymphocyte counts $(13.08 \pm 7.28$ vs. $24.19 \pm 10.87, \mathrm{P}<$ $0.001)$, and higher C-reactive protein (CRP) (5.57 \pm 7.83 vs. $0.93 \pm 1.91, \mathrm{P}<0.001)$. Laboratory data in patients admitted vs. patients not admitted to the hospital are presented in Table 2.

\section{Radiographic studies}

Overall, we obtained chest X-rays in $67.2 \%$ of the patients, abdominal X-rays in $60 \%$, abdominal $\mathrm{U} / \mathrm{S}$ in $46.4 \%$ and abdominal CAT scan in $16.8 \%$. Use of radiologic studies was higher in patients admitted to the hospital, and the difference was significant for chest X-rays (42 of 47 vs. 42 of $78, \mathrm{P}<$ $0.001)$ and for CAT scan of the abdomen ( 15 of 47 vs. 6 of 78, $\mathrm{P}<0.001$ ), whereas differences with regard to X-rays and U/S of the abdomen were not significant. Abnormal findings on radiologic workup were significantly more common in admitted patients ( 35 of 77 vs. 23 of $78, P<0.001$ ).

\section{ED diagnosis and hospital admission}

After evaluation in the ED, 47 patients $(37.6 \%)$ were admitted to the hospital and $78(62.4 \%)$ were not. Depending on diagnostic workup findings, patients were admitted to different services: $25(53.2 \%)$ were admitted in the internal medicine ward, $17(36.2 \%)$ in the surgery ward and five $(10.6 \%)$ in other wards.

Final diagnoses at the time patients left the ED to either be admitted to the hospital or be discharged to home are summarized in Table 3. It is worth pointing that in $13.6 \%$ of cases it was not possible to establish a diagnosis before the patient was discharged from the ED.

\section{Predictive factors for hospital admission}

We considered an elevated WBC if WBCs $>12,000 / \mathrm{mm}^{3}$, an elevated CRP level if CRP $>0.5 \mathrm{mg} / \mathrm{dL}$, abnormal imaging if any abnormal finding in the radiograph, U/S or CAT stud- 
Table 2. Laboratory Findings in Patients Admitted vs. Patients Not Admitted to the Hospital

\begin{tabular}{llll}
\hline Variable & Admitted $(\mathbf{n}=\mathbf{4 7})$ & Not admitted $(\mathbf{n}=\mathbf{7 8})$ & P value \\
\hline WBCs & $14,193 \pm 6,182$ & $9,641 \pm 2,883$ & 0.001 \\
Neutrophils & $79.99 \pm 9.21$ & $67.72 \pm 12.57$ & 0.001 \\
Lymphocytes & $13.08 \pm 7.28$ & $24.19 \pm 10.87$ & 0.001 \\
Mononuclear cells & $5.76 \pm 2.49$ & $5.86 \pm 2.04$ & 0.810 \\
Platelets & $238.9 \pm 69.1$ & $246.9 \pm 51.9$ & 0.474 \\
Hemoglobin & $13.4 \pm 1.8$ & $13.3 \pm 1.3$ & 0.786 \\
Hematocrit & $39.3 \pm 4.6$ & $38.9 \pm 3.4$ & 0.501 \\
INR & $1.24 \pm 1.08$ & $1.04 \pm 0.09$ & 0.166 \\
PTT & $34.43 \pm 4.32$ & $34.58 \pm 3.56$ & 0.844 \\
Serum sodium & $138.2 \pm 3.6$ & $138.9 \pm 2.4$ & 0.276 \\
Serum potassium & $4.2 \pm 0.5$ & $4.2 \pm 0.6$ & 0.990 \\
Serum calcium & $9.62 \pm 0.78$ & $9.74 \pm 0.50$ & 0.388 \\
Serum glucose & $132.0 \pm 65.4$ & $103.8 \pm 25.2$ & 0.019 \\
Serum creatinine & $1.09 \pm 0.81$ & $0.88 \pm 0.21$ & 0.074 \\
SGOT & $29.3 \pm 35.7$ & $25.8 \pm 38.2$ & 0.612 \\
SGPT & $25.5 \pm 24.5$ & $26.7 \pm 57.8$ & 0.896 \\
LDH & $210.3 \pm 119.9$ & $202.1 \pm 59.9$ & 0.650 \\
Amylase & $192.0 \pm 674.6$ & $56.7 \pm 26.3$ & 0.212 \\
CRP & $5.57 \pm 7.83$ & $0.93 \pm 1.91$ & 0.001 \\
Urine WBC & $16.7 \pm 30.8$ & $10.3 \pm 24.4$ & 0.279 \\
Urine RBCs & $10.5 \pm 22.9$ & $11.0 \pm 24.3$ & 0.919 \\
\hline
\end{tabular}

WBCs: white blood cells; INR: international normalized ratio; PTT: partial thromboplastin time; SGOT: serum glutamic oxaloacetic transaminase; SGPT: serum glutamic pyruvic transaminase; LDH: lactate dehydrogenase; CRP: C-reactive protein; RBCs: red blood cells. All values are reported as mean \pm SD. Due to multiple comparisons, only $P$ values $<0.05 / 21$, therefore $\mathrm{P}<0.0023$ are considered significant.

ies and fever if body temperature measured in the axilla was above $37.2^{\circ} \mathrm{C}$. Patients admitted to the hospital had significantly higher age $(51.35 \pm 23.7$ vs. $43 \pm 8.3$ years, $\mathrm{P}<0.03)$, higher WBC $\left(14,194 \pm 6,182 / \mathrm{mm}^{3}\right.$ vs. $9,641 \pm 2,883 / \mathrm{mm}^{3}, \mathrm{P}$ $<0.01)$ and CRP levels $(5.57 \pm 7.8 \mathrm{mg} / \mathrm{dL}$ vs. $0.93 \pm 1.9 \mathrm{mg} /$ $\mathrm{dL}, \mathrm{P}<0.01)$. Frequency of abnormal imaging findings was also significantly higher in admitted patients ( 35 of 77 vs. 23 of $78, \mathrm{P}<0.001)$.

Furthermore, binary logistic regression analysis showed that abnormal imaging findings (odds ratio $(\mathrm{OR})=6.47,95 \%$ confidence interval (CI): $2.11-19.77, \mathrm{P}<0.001)$ and elevated serum CRP levels $(\mathrm{OR}=6.24,95 \% \mathrm{CI}$ : $2.16-18.03, \mathrm{P}<$ $0.001)$ were significant factors predicting hospital admission, whereas elevated WBC count and presence of fever were not significant predictors.

\section{Discussion}

AAP is a common problem in the ED, requires use of hospital resources and significantly contributes to health care cost [5], and patient evaluation can be challenging, because multiple diagnoses need to be considered in a limited time frame, and available information can be inconclusive. Because AAP can be due to a wide range of entities, including infection, mechanical obstruction, malignancy, cardiac problems and gastrointestinal ischemia [6], severity ranges from self-limited symptoms to true surgical emergencies [7]. Patients with AAP represent a diverse group, symptom presentation can differ in certain populations, such as in elderly or immunocompromised patients [8], and diagnosis can be unclear due to atypical history or physical findings. Yet, because the underlying pathology can be life threatening [9-11], physicians often rely on laboratory testing and radiologic imaging to expedite diagnosis [12] in order to initiate appropriate timely therapeutic intervention $[13,14]$.

Identification of warning "red flags" in the history and physical examination, together with appropriate imaging and laboratory studies may help detect patients with a serious underlying disease process $[15,16]$. Although clinicians often withhold pain medication before a diagnosis is established, several studies have demonstrated that use of analgesics does not negatively influence diagnosis or treatment in the ED [15, 17]. In this manuscript, we present the results of a prospective study on patients assessed in the ED of the University Hospital of Patras with abdominal pain as main symptom in the period 
Table 3. Diagnosis After Emergency Department Assessment

\begin{tabular}{|c|c|c|c|c|}
\hline Diagnosis & Cases $(\%)$ & Admitted $(n=47)$ & Not admitted $(n=78)$ & $\mathbf{P}$ \\
\hline Gastroenteritis & $16(12.8)$ & 5 & 11 & 0.783 \\
\hline Gastritis/gastric/duodenal ulcer & $15(12.0)$ & 1 & 14 & 0.009 \\
\hline Gastrointestinal perforation & $3(2.4)$ & 3 & 0 & 0.051 \\
\hline Diverticulitis & $6(4.8)$ & 4 & 2 & 0.196 \\
\hline Ileus & $4(3.2)$ & 3 & 1 & 0.148 \\
\hline Gynecological diagnosis & $6(4.8)$ & 3 & 3 & 0.671 \\
\hline Musculoskeletal pain & $4(3.2)$ & 0 & 4 & 0.296 \\
\hline Nephrolithiasis/colic & $7(5.6)$ & 1 & 6 & 0.253 \\
\hline Constipation & $4(3.2)$ & 0 & 4 & 0.296 \\
\hline Abdominal bloating & $2(1.6)$ & 0 & 2 & 0.519 \\
\hline Cholecystitis & $3(2.4)$ & 3 & 0 & 0.051 \\
\hline Pulmonary embolism & $1(0.8)$ & 1 & 0 & 0.376 \\
\hline GI bleeding & $1(0.8)$ & 1 & 0 & 0.376 \\
\hline Peritoneal metastases & $1(0.8)$ & 1 & 0 & 0.376 \\
\hline Porcelain gallbladder & $1(0.8)$ & 0 & 1 & 1.000 \\
\hline GERD/esophagitis & $1(0.8)$ & 0 & 1 & 1.000 \\
\hline Peritonitis & $1(0.8)$ & 1 & 0 & 0.376 \\
\hline
\end{tabular}

GI: gastrointestinal; GERD: gastroesophageal reflux disease; ED: emergency department.

October 1 through November 30, 2015 and April 15 through May 31, 2016. These periods were selected in order to avoid high tourist seasons and attempt to capture a patient population that reflects the local population.

In accordance with international standards, patients were evaluated in the ED in order to reach a diagnosis, and received treatment aimed at providing pain relief and maintaining hemodynamic stability, as evidenced by adequate urine output and monitoring of vital signs.

Patient assessment was based on history of present illness, past medical, surgical and social history, together with careful clinical examination emphasizing specific physical signs related to underlying pathology of the abdominal cavity and relevant laboratory and radiologic diagnostic evaluation. Although the character and location of abdominal pain were not predictors of hospital admission in our study, specific clinical signs on the abdominal exam were crucial, as evidenced by the fact that $70 \%$ of patients with Murphy sign, $84.6 \%$ with McBurney sign and $88.9 \%$ of patients with rebound tenderness were admitted to the hospital. This fact underlies the importance of careful hands-on clinical examination as part of comprehensive patient evaluation in the ED.

Our study also showed that frequency of normal chest and abdominal X-rays was high both in patients admitted and not admitted to the hospital. Overall, only $33.3 \%$ of abdominal $\mathrm{U} / \mathrm{S}$ and $6.7 \%$ of abdominal CAT scans were normal (revealing no pathology), and these findings are in agreement with earlier published data suggesting that abdominal X-rays have low yield for clinically useful information and are probably overused $[18,19]$. U/S and CAT imaging in patients with certain history and clinical exam findings can probably help clinicians decide whether a patient with abdominal pain should be admitted to the hospital or not.

Limitations of our study include possible inter- and intraexaminer variability, the relatively small sample size, and absence of standardized criteria for admission to the hospital. However, we believe that this study is representative of current clinical practice patterns in this Mediterranean tertiary care academic center. Furthermore, we believe that our findings add useful insights regarding AAP, a problem commonly seen in the ED.

Evaluation of the patient who presents with abdominal pain in the ED remains one of the most important topics in acute medicine. Despite great diagnostic and therapeutic advances, including abdominal CAT, ultrasonography and laparoscopy, misdiagnosis has been reported [20] and it is important to em- 
phasize that all diagnostic studies can have false negative findings. The decision to admit a patient to the hospital without a clear diagnosis or explanation for the pain is matter of debate, and several approaches are considered reasonable. The phrase "treat the patient, not the test" is still very appropriate for patients with AAP, and ongoing patient reassessment in the ED seems to be a reasonable option. Hospital discharge with clear instructions to the patient and family regarding possible onset of new symptoms or clinical deterioration is also acceptable. Limited financial resources can be the reason for limiting expensive diagnostic radiographic studies and expensive diagnostic laboratory tests, such as sepsis biomarkers.

\section{Conclusions}

Appropriate diagnostic evaluation and decision for or against hospitalization is a challenge in the patient who comes to the ED with AAP. Our data from this small prospective clinical trial suggest that increasing abdominal pain intensity, presence of Murphy or McBurney signs, abnormal radiologic findings and elevated CRP are potential predictors of the need for hospitalization. However, because all currently available diagnostic tests can give falsely negative findings, vigilance, together with detailed evaluation of all available data is needed in order to avoid overlooking potentially life-threatening causes of abdominal pain. Larger, well-designed prospective clinical trials are needed to better define predictive factors and establish accepted care paths for this challenging patient population.

\section{Conflict of Interest}

All authors state that they do not have any conflict of interest to report.

\section{Author Contributions}

DV wrote and edited the manuscript, MK contributed to study design, did statistical analysis, revised and finalized the manuscript, NP wrote the manuscript and did statistical analysis, GK collected data, VM collected data, KK collected data, EF collected data, CA collected data, CG supervised and edited the manuscript. All authors have read and approved the manuscript.

\section{Grant Support}

This work was supported solely by department funds.

\section{Abbreviations}

AAP: acute abdominal pain; CDC: Center for Disease Control; CI: confidence interval; CAT: computerized axial tomography;
CRP: C-reactive protein; ED: emergency department; INR: international normalized ratio; LDH: lactate dehydrogenase; OR: odds ratio; PTT: partial thromboplastin time; SGOT: serum glutamic-oxaloacetic transaminase; SGPT: serum glutamic pyruvic transaminase; WBC: white blood cell

\section{References}

1. Gerhardt RT, Nelson BK, Keenan S, Kernan L, MacKersie A, Lane MS. Derivation of a clinical guideline for the assessment of nonspecific abdominal pain: the Guideline for Abdominal Pain in the ED Setting (GAPEDS) Phase 1 Study. Am J Emerg Med. 2005;23(6):709-717.

2. Kamin RA, Nowicki TA, Courtney DS, Powers RD. Pearls and pitfalls in the emergency department evaluation of abdominal pain. Emerg Med Clin North Am. 2003;21(1):61-72, vi.

3. Lewis LM, Banet GA, Blanda M, Hustey FM, Meldon SW, Gerson LW. Etiology and clinical course of abdominal pain in senior patients: a prospective, multicenter study. J Gerontol A Biol Sci Med Sci. 2005;60(8):10711076.

4. Cooper GS, Shlaes DM, Salata RA. Intraabdominal infection: differences in presentation and outcome between younger patients and the elderly. Clin Infect Dis. 1994;19(1):146-148.

5. Myer PA, Mannalithara A, Singh G, Singh G, Pasricha PJ, Ladabaum U. Clinical and economic burden of emergency department visits due to gastrointestinal diseases in the United States. Am J Gastroenterol. 2013;108(9):14961507.

6. Dang C, Aguilera P, Dang A, Salem L. Acute abdominal pain. Four classifications can guide assessment and management. Geriatrics. 2002;57(3):30-32, 35-36, 41-32.

7. Cartwright SL, Knudson MP. Evaluation of acute abdominal pain in adults. Am Fam Physician. 2008;77(7):971978.

8. Lyon C, Clark DC. Diagnosis of acute abdominal pain in older patients. Am Fam Physician. 2006;74(9):15371544.

9. McNamara R, Dean AJ. Approach to acute abdominal pain. Emerg Med Clin North Am. 2011;29(2):159-173, vii.

10. Flasar MH, Cross R, Goldberg E. Acute abdominal pain. Prim Care. 2006;33(3):659-684, vi.

11. Macaluso CR, McNamara RM. Evaluation and management of acute abdominal pain in the emergency department. Int J Gen Med. 2012;5:789-797.

12. Mills AM, Dean AJ, Hollander JE, Chen EH. Abdominal pain: a survey of clinically important outcomes for future research. CJEM. 2010;12(6):485-490.

13. Chiche L, Roupie E, Delassus P. [Management of adult abdominal pain in the Emergency Room]. J Chir (Paris). 2006;143(1):6-14.

14. Trentzsch H, Werner J, Jauch KW. [Acute abdominal pain in the emergency department - a clinical algorithm for adult patients]. Zentralbl Chir. 2011;136(2):118-128.

15. Abbas SM, Smithers T, Truter E. What clinical and labo- 
ratory parameters determine significant intra abdominal pathology for patients assessed in hospital with acute abdominal pain? World J Emerg Surg. 2007;2:26.

16. Stone R. Primary care diagnosis of acute abdominal pain. Nurse Pract. 1996;21(12 Pt 1):19-20, 23-30, 35-19; quiz 40-11.

17. Falch C, Vicente D, Haberle H, Kirschniak A, Muller S, Nissan A, Brucher BL. Treatment of acute abdominal pain in the emergency room: a systematic review of the literature. Eur J Pain. 2014;18(7):902-913.
18. Jackson K, Taylor D, Judkins S. Emergency department abdominal x-rays have a poor diagnostic yield and their usefulness is questionable. Emerg Med J. 2011;28(9):745749.

19. Sreedharan S, Fiorentino M, Sinha S. Plain abdominal radiography in acute abdominal pain - is it really necessary? Emerg Radiol. 2014;21(6):597-603.

20. Flum DR, Morris A, Koepsell T, Dellinger EP. Has misdiagnosis of appendicitis decreased over time? A population-based analysis. JAMA. 2001;286(14):1748-1753. 